\title{
Applications of an isogeometric indirect boundary element method and the importance of accurate geometrical representation in acoustic problems
}

\author{
Emin Oguz Inci ${ }^{\mathrm{a}, \mathrm{b}, 1}$, Laurens Coox $^{\mathrm{a}, \mathrm{b}}$, Onur Atak $^{\mathrm{c}}$, Elke Deckers $^{\mathrm{a}, \mathrm{b}}$, Wim \\ Desmet $^{\mathrm{a}, \mathrm{b}}$ \\ ${ }^{a} K U$ Leuven, Department of Mechanical Engineering, Celestijnenlaan 300, B-3001, \\ Leuven, Belgium \\ ${ }^{b}$ DMMS lab, Flanders Make \\ ${ }^{c}$ Siemens Industry Software NV, Interleuvenlaan 68, B-3001, Leuven, Belgium
}

\begin{abstract}
This study presents the performance and the potential use of an isogeometric indirect variational boundary element method (IGiBEM) for industrial acoustics applications. The method is validated against semi-analytical frequency response solutions for a basic problem. After being validated, the performance of the IGiBEM is tested and benchmarked against the conventional indirect boundary element method (iBEM) for geometrically complex industrial applications. A vibrating car hood and a loudspeaker model with a vibrating woofer are used as industrial numerical tests. A non-isoparametric iBEM is included in the benchmarks to distinguish the contribution of the discretization versus the geometrical errors to overall error. The tests indicate that the geometrical errors play a more important role in problems involving complex geometries and high-frequency excitations. The study also demonstrates the advantages of IGiBEM over the conventional iBEM regarding the accuracy and computational efficiency for geometrically complex problems.
\end{abstract}

Keywords: Isogeometric Analysis, Indirect Boundary Element Method, Numerical Acoustics, Industrial Acoustics Applications

\footnotetext{
${ }^{1}$ Corresponding author. E-mail address: eminoguz.inci@kuleuven.be
} 


\section{Introduction}

The virtual development cycle starts with computer-aided design (CAD). The CAD model constitutes the geometrical properties of a product. The CAD geometry needs to be discretized to be analysis-suitable. This meshing step is a time-consuming process which also adds a new link to the optimization chain of the product. Any modification in the design geometry requires another meshing process for the analysis of the new model. Moreover, fully automated meshing of the product is rarely achievable and requires additional observation and editing by the engineers. Therefore, the process counts up to $80 \%$ of the overall analysis time [1]. Additionally, meshing means that the CAD geometry is replaced by an approximation to be used in the analysis. As a result, the exact geometry is not used in the simulations. Although it is an advantage for some cases involving too detailed geometry for the engineering problem, it sometimes causes crucial inaccuracies in the simulation results, especially in the problems requiring precision in geometrical surface representation.

A model which constitutes all the design- and analysis-related information is a vision for the computer-aided engineering (CAE). Following this vision, some meshfree methods have been developed in the early 1980's [2]. The idea behind this development is to avoid generating a new analysis-suitable geometry, but instead operating on the points selected on the continuum domain. However, due to performance issues and extra efforts to set essential boundary conditions, the meshfree methods are not prominently used in the industry [3] [4. In 2005, Hughes et al. developed a new method called isogeometric analysis (IGA) to integrate FEA with CAD [1]. The method uses the shape functions and connectivity information of the Non-Uniform Rational B-Splines (NURBS), T-Splines, subdivision surfaces or other types of surface representation functions in the CAD files. After the introduction, IGA attracted much attention in the scientific community. The method is successfully applied to many engineering applications, such as structural mechanics [1, 5, 6], contact and fracture mechanics [7, 8, 9], fluid mechanics [10, fluid-structure interaction [11] and acoustics [12, 13, 14].

The isogeometric boundary element method poses a huge potential, as the geometrical representation of the solid design models is commonly the boundary representation of the surfaces, instead of volumes. The disadvantage of constructing a volumetric NURBS formulation from a boundary representation, therefore, vanishes for the boundary element method (BEM). 
This makes the isogeometric boundary element method highly suitable to solve acoustic problems. RN Simpson et al. first applied the IGA method to acoustics [12]. A direct collocational BEM approach using isogeometric analysis combined with T-Splines was applied to the time-harmonic acoustic problems. However, a direct BEM formulation requires an enclosed surface and can only solve for either the interior or exterior domains. To be more flexible on geometries to be addressed, an isogeometric indirect variational boundary element method which can solve combined interior/exterior domains, i.e. open boundary problems, was developed by Coox et al. [13].

This paper presents industrial demonstrations of the isogeometric indirect variational boundary element method developed by Coox et al. The numerical examples illustrate the influence of the geometrical error in complex acoustic simulations at low and high-frequency ranges and the advantages of the proposed method against the conventional boundary element methods to eliminate such error. The paper is organized as follows: Section 2 describes the mathematical formulation used for the acoustics problem. Section 3 gives the fundamentals of the isogeometric indirect boundary element method. In section 4, the method is validated with basic numerical examples and the effect of the initial parameters. Section 5 illustrates the accuracy and the performance of the method is benchmarked against the conventional BEM on acoustics problems involving complex geometries. In the last section, conclusions, limitations and further research are discussed.

\section{Problem formulation}

The problem under study is finding the steady-state acoustic pressure at any point in an unbounded fluid domain given the boundary conditions on a surface. The steady-state acoustic pressure, $p$, at any point in an unbounded domain $\Omega$ filled with a fluid at rest with uniform density $\rho$ due to an external source distribution $q$ at an angular frequency $\omega$ is governed by the inhomogeneous Helmholtz equation,

$$
\nabla^{2} p(\mathbf{r})+k^{2} p(\mathbf{r})=-j \rho \omega q \delta\left(\boldsymbol{r}, \boldsymbol{r}_{\boldsymbol{q}}\right), \quad \mathbf{r} \in \Omega
$$

where $k=\omega / c=2 \pi f / c$ is the acoustic wavenumber, $c$ is the speed of sound, $q$ is the strength of an acoustic volume velocity source at a position $r_{q}$. The mathematical terms used above are as follows: $j^{2}=-1$ is the imaginary unit, $\delta(i, j)$ denotes the Dirac-delta function, $\nabla^{2}=\nabla \cdot \nabla=\frac{\partial^{2} \cdot}{\partial x^{2}}+\frac{\partial^{2} \cdot}{\partial y^{2}}+\frac{\partial^{2} \text {. }}{\partial z^{2}}$ denotes the Laplace operator. 


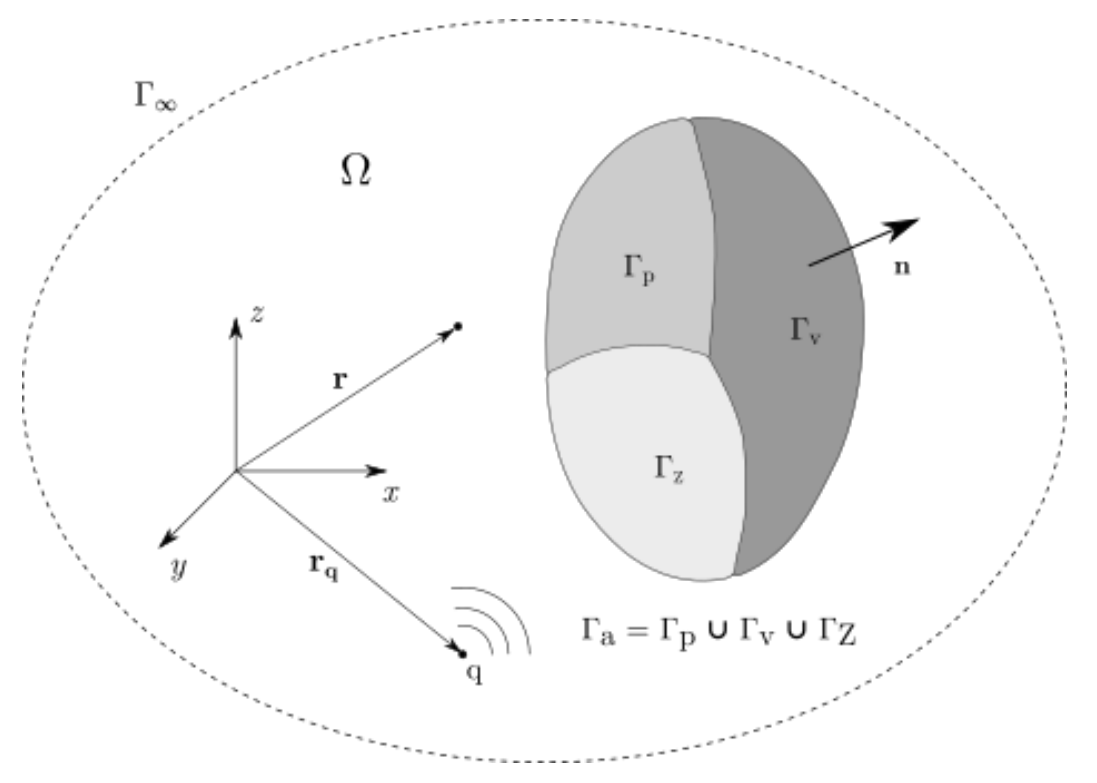

Figure 1: Description of a general unbounded acoustic problem in three dimensions.

In order to obtain a unique solution for the primary variables on the surfaces of the boundary element model, each boundary element must be given a specific boundary condition. There are two distinct boundaries for unbounded domains: (i) $\Gamma_{a}=\Gamma_{p} \cap \Gamma_{v} \cap \Gamma_{Z}$ the finite bounded surface boundary, (ii) $\Gamma_{\infty}$ the fictitious boundary at infinity. Three types of boundary conditions could be imposed to the finite boundary of the fluid domain. The Dirichlet, Neumann and Robin boundary conditions are defined by, respectively (2a), (2b), (2c),

$$
\begin{aligned}
p(\boldsymbol{r}) & =\bar{p}(\boldsymbol{r}), & \boldsymbol{r} & \in \Gamma_{p}, \\
\frac{j}{\rho \omega} \frac{\partial p}{\partial n} & =\bar{v}_{n}(\boldsymbol{r}), & \boldsymbol{r} & \in \Gamma_{v}, \\
\frac{j}{\rho \omega} \frac{\partial p}{\partial n} & =\frac{p(\boldsymbol{r})}{\bar{Z}_{n}}, & \boldsymbol{r} & \in \Gamma_{Z},
\end{aligned}
$$

where $n$ is the unit normal vector pointing away from the fluid domain on the finite boundary. The parameters $\bar{p}, \bar{v}_{n}, \bar{Z}_{n}$ denote the prescribed sound pressure, the prescribed normal velocity perturbation, and the prescribed normal impedance, respectively. 
Apart from the boundary conditions on the finite boundary, Sommerfeld's radiation condition must be satisfied at infinity, $\Gamma_{\infty}$. This boundary condition ensures that no energy is reflected back to the fluid domain. This boundary condition is formulated as [15]

$$
\lim _{\|\boldsymbol{r}\| \rightarrow \infty} \mathbf{r}\left(\frac{\partial p}{\partial \mathbf{r}}+j k p(\mathbf{r})\right)=0
$$

\section{Isogeometric indirect variational boundary element method}

\subsection{Indirect variational boundary element method}

The boundary element method is ideal for acoustic problems with unbounded domains because the formulation intrinsically satisfies Sommerfeld's radiation condition. Not only the exterior problems could be solved, the BEM is able to simulate interior problems as well. By the use of Green's second identity, BEM solves for the primary variables on the fluid domain by means of discretizing the boundary only, unlike the finite element method (FEM). This is a big relief for the meshing stage as there is no need for the volume discretization. The dimension of the problem is decreased by one, and therefore, it leads to a significant decrease in the number of degrees of freedom to be solved for. However, the resulting system matrices are fully populated, complex, and frequency-dependent which hampers the efficiency of the method for large problems. Various acceleration techniques exist for such cases, e.g. Fast Multipole BEM [16, 17], BEM with H-matrices [18], however, they are not the focus of the current study.

The two most common BEM formulations for computational engineering problems are: (i) Direct collocational approach and (ii) indirect variational approach. The collocational BEM solves the strong formulation of the governing equation at the collocation points chosen to represent the boundary of the domain [15]. The unknowns are direct physical values, i.e. pressure or velocity. The direct boundary element method (ABEM) can solve the problems involving either only interior(bounded) or exterior(unbounded) domains. Moreover, it leads to non-symmetric matrices which are more time consuming to solve and requires twice the memory. The indirect variational boundary element method uses a weak formulation instead of solving the strong form in a set of points, therefore, it results in a double surface integral. While the dBEM approach can handle only the problems with a closed boundary surface, the iBEM can also handle open boundaries, thus, 
combined interior/exterior problems. To be able to solve a broader class of applications, this study focuses on the indirect variational boundary element method.

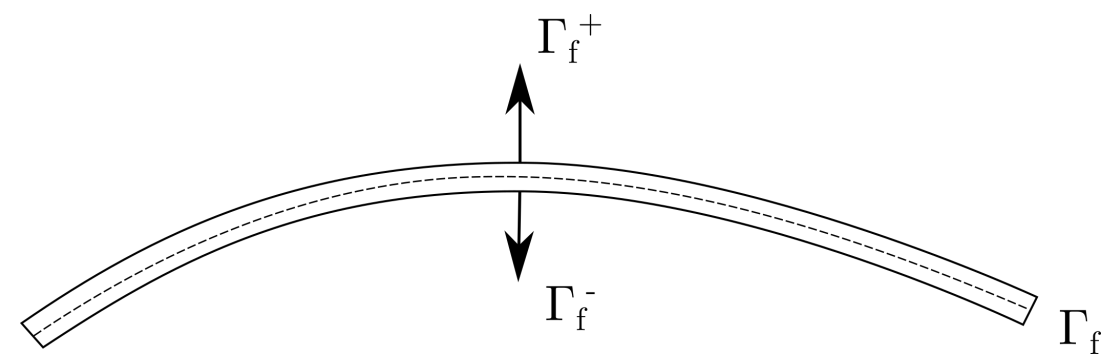

Figure 2: A 2D open boundary illustration for the indirect boundary integral formulation.

\subsubsection{The indirect boundary integral formulation}

The indirect variational boundary element method solves the acoustic pressure or velocity difference between the two sides of the boundary instead of the acoustic pressure. In order to assume a surface as an open-boundary, the surface thickness must be negligible compared to other dimensions, e.g. shells or plates. Otherwise, the boundary should be modelled as a closed boundary of a volume. An open-boundary is the special case of a closed boundary of which two sides are represented with a single surface, see Fig. 2. The primary and secondary variables are the double layer potential, $\mu\left(\mathbf{r}_{f}\right)$, and the single layer potential, $\sigma\left(\mathbf{r}_{f}\right)$ defined on the shared surface as

$$
\begin{array}{rlrl}
\mu\left(\mathbf{r}_{f}\right) & =p\left(\mathbf{r}_{f}^{+}\right)-p\left(\mathbf{r}_{f}^{-}\right) & \mathbf{r}_{f} \in \Gamma_{f} \\
\sigma\left(\mathbf{r}_{f}\right)=\frac{\partial p\left(\mathbf{r}_{f}^{+}\right)}{\partial n}-\frac{\partial p\left(\mathbf{r}_{f}^{-}\right)}{\partial n} & \mathbf{r}_{f} \in \Gamma_{f}
\end{array}
$$

where $\mathbf{r}_{f}$ represents a position at the open-boundary surface. Then, these variables are inserted into boundary integral formulation:

$$
p(\mathbf{r})=\int_{\Gamma_{f}}\left(\mu\left(\mathbf{r}_{f}\right) \frac{\partial G\left(\mathbf{r}, \mathbf{r}_{\mathbf{f}}\right)}{\partial \mathbf{n}}-\sigma\left(\mathbf{r}_{f}\right) G\left(\mathbf{r}, \mathbf{r}_{\mathbf{f}}\right)\right) d \Gamma_{f} \quad \forall \mathbf{r} \in \Omega \backslash \Gamma_{f}
$$

$G\left(\mathbf{r}, \mathbf{r}_{\mathbf{f}}\right)$ represents the Green's kernel function which is the impulse response of the inhomogeneous Helmholtz equation at a point, $\mathbf{r}_{f}$ on the sur- 
face, where $\mathbf{r}$ represents the location of the impulse. The Green's kernel function for three dimensional space problems is formulated as follows:

$$
G\left(\mathbf{r}, \mathbf{r}_{\mathbf{f}}\right)=\frac{e^{-j k\left\|\mathbf{r}-\mathbf{r}_{\mathbf{f}}\right\|}}{4 \pi\left\|\mathbf{r}-\mathbf{r}_{\mathbf{f}}\right\|}
$$

As discussed in section 2, each boundary element must be given a single specific boundary condition. This means that there must be only a single independent variable along an element surface. As primary variables change according to the indirect formulation, the boundary conditions need to be revised. The indirect formulation of the Dirichlet, Neumann and Robin boundary conditions can be defined as below assuming the impedance, $\bar{Z}^{+}=\bar{Z}^{-}=\rho c / \beta$, respectively.

$$
\begin{aligned}
\mu\left(\mathbf{r}_{f}\right) & =0, & & \mathbf{r}_{f} \in \Gamma_{p} \\
\sigma\left(\mathbf{r}_{f}\right) & =0, & & \mathbf{r}_{f} \in \Gamma_{v} \\
\sigma\left(\mathbf{r}_{f}\right) & =-j k \bar{\beta}\left(\mathbf{r}_{f}\right) \mu\left(\mathbf{r}_{f}\right), & & \mathbf{r}_{f} \in \Gamma_{Z}
\end{aligned}
$$

The boundary condition formulations show that either single or double layer potential is zero or they are related to each other at each boundary element. The partition of the boundary to different boundary conditions transforms Eq. (5) into:

$$
\begin{aligned}
p(\mathbf{r})= & -\int_{\Gamma_{p}} \sigma\left(\mathbf{r}_{f}\right) G\left(\mathbf{r}, \mathbf{r}_{f}\right) d \Gamma_{p}+\int_{\Gamma_{v}} \mu\left(\mathbf{r}_{f}\right) \frac{\partial G\left(\mathbf{r}, \mathbf{r}_{\mathbf{f}}\right)}{\partial \mathbf{n}} d \Gamma_{v} \\
& +\int_{\Gamma_{Z}} \mu\left(\mathbf{r}_{f}\right)\left(\frac{\partial G\left(\mathbf{r}, \mathbf{r}_{f}\right)}{\partial \mathbf{n}}+j k \beta\left(\mathbf{r}_{f}\right) \bar{G}\left(\mathbf{r}, \mathbf{r}_{f}\right)\right) d \Gamma_{Z} \quad \forall \mathbf{r} \in \Omega \backslash \Gamma_{f}
\end{aligned}
$$

Galerkin's method is applied to the boundary residuals to obtain the variational formulation.

$$
\begin{array}{r}
\int_{\Gamma_{p}} R_{p}(\sigma, \mu, \mathbf{r}) \delta \sigma d \Gamma_{p}\left(\mathbf{r}+\int_{\Gamma_{v}} R_{v}(\sigma, \mu, \mathbf{r}) \delta \mu d \Gamma_{v}(\mathbf{r})\right. \\
+\int_{\Gamma_{Z}} R_{Z}(\sigma, \mu, \mathbf{r}) \delta \mu d \Gamma_{Z}(\mathbf{r})=0, \quad \forall(\delta \sigma, \delta \mu)
\end{array}
$$

$R_{p}, R_{v}$ and $R_{Z}$ are the boundary residuals for Dirichlet, Neumann, and Robin boundaries conditions, respectively. 
The mathematical expansion of the residuals are as follows:

$$
\begin{aligned}
& R_{p}=-\int_{\Gamma_{p}} \sigma\left(\mathbf{r}_{f}\right) G\left(\mathbf{r}, \mathbf{r}_{f}\right) d \Gamma_{p}\left(\mathbf{r}_{f}\right)+\int_{\Gamma_{p}} \mu(\mathbf{r}) \frac{\partial G\left(\mathbf{r}, \mathbf{r}_{f}\right)}{\partial n\left(\mathbf{r}_{f}\right)} d \Gamma_{v}\left(\mathbf{r}_{f}\right) \\
& +\int_{\Gamma_{Z}} \mu\left(\mathbf{r}_{f}\right)\left(\frac{\partial G\left(\mathbf{r}, \mathbf{r}_{f}\right)}{\partial n\left(\mathbf{r}_{f}\right)}+j k \bar{\beta}\left(\mathbf{r}_{f}\right) G\left(\mathbf{r}, \mathbf{r}_{f}\right)\right) d \Gamma_{v}\left(\mathbf{r}_{f}\right)-\bar{p}(\mathbf{r}) \\
& R_{v}=-\int_{\Gamma_{p}} \sigma\left(\mathbf{r}_{f}\right) \frac{\partial G\left(\mathbf{r}, \mathbf{r}_{f}\right)}{\partial n(\mathbf{r})} d \Gamma_{p}\left(\mathbf{r}_{f}\right)+\int_{\Gamma_{v}} \mu\left(\mathbf{r}_{f}\right) \frac{\partial^{2} G\left(\mathbf{r}, \mathbf{r}_{f}\right)}{\partial n(\mathbf{r}) \partial n\left(\mathbf{r}_{f}\right)} d \Gamma_{v}\left(\mathbf{r}_{f}\right) \\
& +\int_{\Gamma_{Z}} \mu\left(\mathbf{r}_{f}\right)\left(\frac{\partial^{2} G\left(\mathbf{r}, \mathbf{r}_{f}\right)}{\partial n(\mathbf{r}) \partial n\left(\mathbf{r}_{f}\right)}+j k \bar{\beta}\left(\mathbf{r}_{f}\right) \frac{\partial G\left(\mathbf{r}, \mathbf{r}_{f}\right)}{\partial n(\mathbf{r})}\right) d \Gamma_{Z}\left(\mathbf{r}_{f}\right)+j \rho \omega \bar{v}_{n}(\mathbf{r}) \\
& R_{Z}=-\int_{\Gamma_{p}} \sigma\left(\mathbf{r}_{f}\right) \frac{\partial G\left(\mathbf{r}, \mathbf{r}_{f}\right.}{\partial n(\mathbf{r})} d \Gamma_{p}\left(\mathbf{r}_{f}\right)+\int_{\Gamma_{v}} \mu\left(\mathbf{r}_{f}\right) \frac{\partial^{2} G\left(\mathbf{r}, \mathbf{r}_{f}\right)}{\partial n(\mathbf{r}) \partial n\left(\mathbf{r}_{f}\right)} d \Gamma_{v}\left(\mathbf{r}_{f}\right) \\
& +\int_{\Gamma_{Z}} \mu\left(\mathbf{r}_{f}\right)\left(\frac{\partial^{2}\left(\mathbf{r}, \mathbf{r}_{f}\right.}{\partial n(\mathbf{r}) \partial n\left(\mathbf{r}_{f}\right)}+j k \bar{\beta}\left(\mathbf{r}_{f}\right) \frac{\partial G\left(\mathbf{r}, \mathbf{r}_{f}\right.}{\partial n(\mathbf{r})}\right) d \Gamma_{Z}\left(\mathbf{r}_{f}\right) \\
& -\int_{\Gamma_{p}} j k \bar{\beta}(\mathbf{r}) \sigma\left(\mathbf{r}_{f}\right) G\left(\mathbf{r}, \mathbf{r}_{f}\right) d \Gamma_{p}\left(\mathbf{r}_{f}\right)+\int_{\Gamma_{v}} j k \bar{\beta}(\mathbf{r}) \mu\left(\mathbf{r}_{f}\right) \frac{\partial G\left(\mathbf{r}, \mathbf{r}_{f}\right)}{\partial n\left(\mathbf{r}_{f}\right)} d \Gamma_{v}\left(\mathbf{r}_{f}\right) \\
& +\int_{\Gamma_{Z}} \mu\left(\mathbf{r}_{f}\right)\left(j k \bar{\beta} \frac{\partial G\left(\mathbf{r}, \mathbf{r}_{f}\right.}{\partial n\left(\mathbf{r}_{f}\right.}-k^{2} \bar{\beta}(r) \bar{\beta}\left(\mathbf{r}_{f}\right) G\left(\mathbf{r}, \mathbf{r}_{f}\right)\right) d \Gamma_{Z}\left(\mathbf{r}_{f}\right)
\end{aligned}
$$

The asymptotic complexity of the boundary element method formulated here is $O\left(N^{3}\right)$ due to dense system matrix.

\subsubsection{Singularity treatment}

When the residual terms are substituted into the variational equation in Eq. (11), they form double integrals. The double integrals contain hypersingularities. The transformation of the hyper-singular terms to weakly singular terms is described in [19]. After the transformation, the formulation is still prone to singularities due to $1 / \mathbf{r}$ term in the Green's function. This can pose a problem in the assembly when two integration points are significantly close to each other, as Gaussian quadrature is used element-by-element for the numerical integration in this study. Sauter and Schwab [20] introduced a way to increase the number of integration points in singularity-critical regions and arrange them in a way to prevent the singularity effects. The 
auto-singularity, which is the integration of identical panels to calculate the effect of the vibration of an element to element itself, and edge-singularity, which is the integration of neighbouring elements which share a common edge, are taken into consideration in this study. The singularity treatment for the elements sharing a common vertex is omitted.

\subsection{Isogeometic Approach and NURBS as Basis Functions}

Isogeometric analysis is a method introduced by Hughes et al. [1] which is based on an idea of using the connectivity information and basis functions available in a CAD model for the numerical analysis in the computational mechanics field. Instead of several meshless methods, such as subdivision surfaces, finite cell methods, using Non-uniform Rational B-Splines (NURBS) attracted more attention by the researchers as NURBS is the standard output of the general CAD file formats, like IGES and STEP. In this section, a brief introduction to NURBS surfaces will be given as this study uses NURBSbased boundary representation models for acoustic analysis.

\subsubsection{Non-uniform rational B-Splines (NURBS)}

NURBS are the piecewise non-periodic rational functions parametrizing curves, surfaces and even volumes. NURBS is a variation of general BSplines. The biggest advantage to use NURBS is the ability to define complex shapes with a small number of control points, therefore with fewer data in Computer Aided Design and computer graphic files. As the NURBS are built from B-Splines, first a brief introduction to B-Splines is given.

B-Splines are the piecewise polynomial functions which are used primarily for parametrizing curves [21]. They consist of several sets of information:

- polynomial degree, $p$

- $n$ control points, $\mathbf{P}_{i}, i=1, \ldots, n$

- knot vector, $\Xi=\left[\xi_{1}, \xi_{2}, \ldots, \xi_{n+p+1}\right]$

- basis functions for each control point, $N_{i}, i=1, \ldots, n$

The polynomial degree, the control points and the basis functions have a common ground with the classical finite element approach where they have similar concepts. However, the knot vector is an unparalleled concept in IGA or B-Splines. The knot vector, $\Xi$, is a set of parametric coordinates, $\xi_{i}$, in a non-decreasing order. This vector represents how a NURBS patch is divided 
into sections, in this case, elements. The spacing between these knots can be adjusted equally, uniform B-Splines, or disproportionately, non-uniform B-Splines. The non-uniformity gives flexibility to the B-Splines to represent more complex shapes. For B-Splines formed by $n$ control points, $n+p+1$ knots are required: $\xi \in \mathbb{R}(k=1, \ldots, n+p+1)$. A single NURBS patch extracted from a CAD file is formed by an open knot vector, where the first and the last unique knot value have a multiplicity of $p+1$.

B-Spline basis functions are defined recursively by Cox-de Boor algorithm [22] [23]:

$$
\begin{array}{rr}
p=0: & N_{i}^{0}(\xi)= \begin{cases}1, & \text { if } \xi_{i} \leqslant \xi \leqslant \xi_{i+1}, \\
0, & \text { else }\end{cases} \\
p>0: & N_{i}^{p}(\xi)=\frac{\xi-\xi_{i}}{\xi_{i+p}-\xi_{i}} N_{i}^{p-1}(\xi)+\frac{\xi_{i+p+1}-\xi}{\xi_{i+p+1}-\xi_{i+1}} N_{i+1}^{p-1}(\xi) .
\end{array}
$$

The B-Spline basis functions have non-negative values in the interval $\left[\xi_{i}, \xi_{i+p+1}\right]$ which constitutes a local support. The basis functions are $C^{\infty}$ continuous inside a knot span and $C^{p-1}$ continuous along single knots. For knots with multiplicity $k$, the continuity of the basis functions reduce to $C^{p-k}$. The basis functions also form partition of unity, $\sum_{i=1}^{n} N_{i, p}(\xi)=1$.

To obtain NURBS basis functions from the B-Spline basis functions, a weight $w_{i}$ is assigined to every B-Spline function $N_{i}^{p}(\xi)$. The NURBS basis functions with polynomial order $\mathrm{p}, R_{i}^{p}(\xi)$ are given as

$$
R_{i}^{p}(\xi)=\frac{N_{i}^{p}(\xi) w_{i}}{\sum_{i=1}^{n} N_{i}^{p}(\xi) w_{i}} \text { with } i=1, \ldots, n .
$$

As the terms in nominator and denominator are both piecewise polynomials, $R_{i}^{p}(\xi)$ is a piecewise rational function. One can also observe that if all the weights are equal, then $R_{i}^{p}(\xi)=N_{i}^{p}(\xi)$.

A NURBS curve, see Fig. 4a, is formed by univariate NURBS basis functions multiplied with associated control point $P_{i}$ :

$$
\mathbf{C}(\xi)=\sum_{i=1}^{n} \frac{N_{i}^{p}(\xi) w_{i} \mathbf{P}_{i}}{\sum_{k=1}^{n} N_{i}^{p}(\xi) w_{k}}=\sum_{i=1}^{n} R_{i}^{p}(\xi) \mathbf{P}_{i}
$$

To establish NURBS surfaces, see Fig. 4b, bivariate NURBS basis functions must be obtained first by the tensor product of the univariate NURBS 


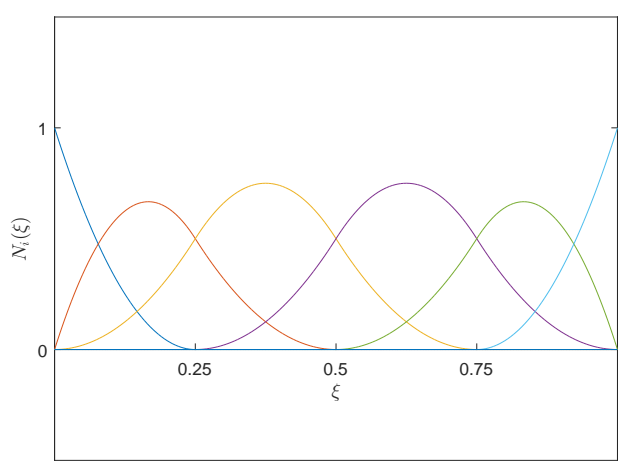

(a) Quadratic NURBS basis functions in 1D

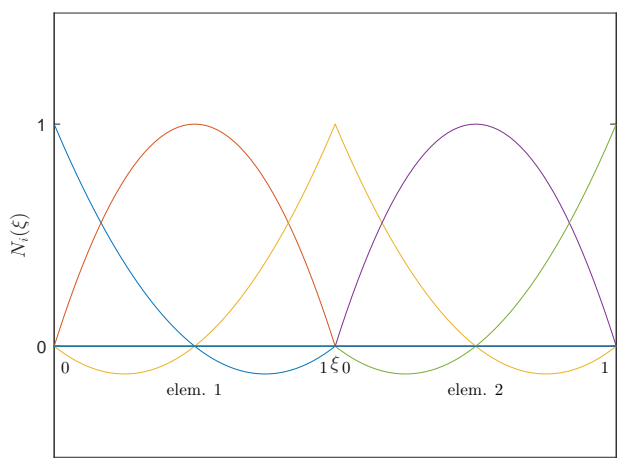

(b) Quadratic Lagrangian basis functions in 1D

Figure 3: A comparison of NURBS and Lagrangian basis function in one dimensional parent domain

bases. Given the polynomial orders, $\mathrm{p}$ and $\mathrm{q}$, and the knot vectors, $\boldsymbol{\Xi}=$ $\left[\xi_{1}, \ldots, \xi_{n+p+1}\right]$ and $\mathbf{H}=\left[\eta_{1}, \ldots, \eta_{m+q+1}\right]$ of the indices of independent univariate NURBS basis functions, bivariate NURBS basis functions is defined as

$$
R_{i, j}^{p, q}(\xi, \eta)=\frac{N_{i}^{p}(\xi) M_{j}^{q}(\eta) w_{i}, j}{\sum_{i=1}^{n} \sum_{j=1}^{m} N_{i}^{p}(\xi) M_{j}^{q}(\eta) w_{i}, j} \text { with } i=1, \ldots, n ; j=1, \ldots, m
$$

where $N_{i}^{p}(\xi)$ and $M_{j}^{p}(\eta)$ are the univariate NURBS basis functions. Then, one can obtain a NURBS surface by multiplying the bivariate bases with their associated control points, $\mathbf{P}_{i, j}$.

$$
S(\xi, \eta)=\sum_{i=1}^{n} \sum_{j=1}^{m} R_{i, j}^{p, q}(\xi, \eta) \mathbf{P}_{i, j}
$$

\subsubsection{NURBS-based isogeometric boundary element method}

In the variational formulation, the boundary residual integrals in Eq. (11) are solved by integration on the discretized elements. In the proposed method, the identical NURBS basis functions which are used for geometry mapping approximate the field in an isoparametric concept. The approximated single and double layer potentials, $\sigma\left(\mathbf{r}_{f}\right)$ and $\mu\left(\mathbf{r}_{f}\right)$, are represented as 


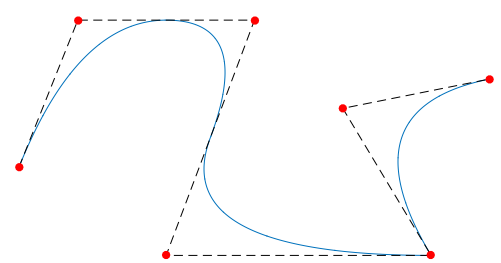

(a) NURBS Curve

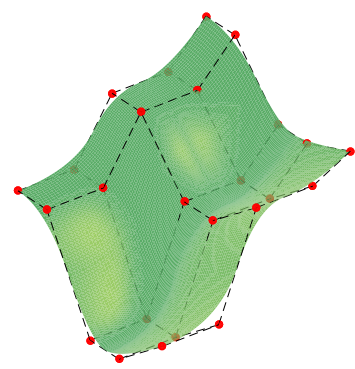

(b) NURBS Surface

Figure 4: An illustration of quadratic NURBS geometries (control points in red) 24]

$$
\begin{array}{ll}
\sigma\left(\mathbf{r}_{f}\right) \approx \sigma^{h}\left(\mathbf{r}_{f}\right)=\sum_{i=1}^{n} N_{i}\left(\mathbf{r}_{f}\right) d_{i}^{\sigma} & \mathbf{r}_{f} \in \Gamma_{\sigma} \\
\mu\left(\mathbf{r}_{f}\right) \approx \mu^{h}\left(\mathbf{r}_{f}\right)=\sum_{i=1}^{n} N_{i}\left(\mathbf{r}_{f}\right) d_{i}^{\mu} & \mathbf{r}_{f} \in \Gamma_{\mu}
\end{array}
$$

where $n$ is the number of NURBS basis functions which is equal to the number of control points and $N_{i}\left(\mathbf{r}_{f}\right)$ is the associated basis function with contribution factors at each control point, $d_{i}^{\bullet}$. Since the NURBS basis functions are not necessarily interpolatory as it is the case for Lagrangian basis functions, the contribution factors at each control point, $d_{i}^{\bullet}$, do not represent physical quantities.

It is also important to satisfy the zero jump of pressure at the free edges of the open boundary,

$$
\mu\left(\mathbf{r}_{f}\right)=0 \quad r_{f} \in \Gamma_{\text {free }}
$$

by equating the double layer potential contribution factors, $d_{i}^{\mu}$ to zero at the control points forming the free edges.

By introducing the equations (21) and (22) into (11), a symmetric global system of equations can be assembled. After the solution of linear system of equations and obtaining $d_{i}^{\sigma}$ and $d_{i}^{\mu}$ values, NURBS basis functions are again 
used to project the approximated single, $\sigma^{h}\left(\mathbf{r}_{f}\right)$, and double layer potentials, $\mu^{h}\left(\mathbf{r}_{f}\right)$ on the boundary surface. The pressure at any point in the fluid domain is computed by post-processing the single and double layer potentials into the boundary integral equation (10).

\subsubsection{Strong patch coupling}

Complex geometries require a geometrical model with multiple patches. The NURBS patches begin and end with an open knot with $C^{-1}$-continuity as default. This violates the requirement to obtain a continuous solution at the patch interfaces because the solution of an inhomogeneous Helmholtz problem by a variational formulation requires $C^{0}$-continuity throughout the domain [13], [25]. Therefore, a weak or strong coupling is necessary to ensure the continuity of the solution at the patch interfaces. A strong patch coupling method developed by Coox et al. 25] is used to avoid discontinuities at the calculations of potentials and to ensure convergence. The method couples the interfaces between matching non-conforming patches with $C^{0}$-continuity.

\subsubsection{Advantages and drawbacks of using IGA over conventional BEM}

The biggest advantage of using the NURBS-based isogeometric indirect boundary element method (IGiBEM) over the conventional boundary element method is the ability to use the CAD geometry directly in the analysis, thus, eliminating the meshing stage, the conversion of the geometry to a simplified analysis-suitable geometry. This does not only reduce the designto-analysis time but also enables the user to solve on the exact geometrical description with CAD. Using the exact geometry is especially important for BEM as only the boundary is discretized and the free field is derived from the potentials obtained at the boundary. The problem defined in Sec. 2 is a frequency domain problem. Therefore, a good representation of geometry plays an important role especially at high frequencies at which wavenumber is greater.

The non-interpolatory nature of the NURBS basis provides a higher continuity over the element intersections. In figure 3, the neighbouring elements are $C^{2}$-continuous for the quadratic NURBS basis functions whereas the Lagrangian basis functions are always interpolatory, $C^{0}$-continous, on element intersections. The continuity within the elements is defined by the polynomial order of the basis function. In order to increase the field order by one, an additional DOF must be introduced to each element with Lagrangian basis functions. On the other hand, $k-1$ DOFs are added to a NURBS 
curve, which consists of many elements, where $k$ represents the number of unique knots on the NURBS curve. As the number of unique knots is equal or smaller than the number of control points, which are equal to the number of DOFs in NURBS curves, a higher continuity is obtained by NURBS basis functions over the elements than Lagrangian basis functions with fewer DOFs.

Another huge advantage of NURBS as basis functions is the ease and richness of refinement in the pre-processing stage while keeping the geometry intact. New knots could be inserted to the index space, $\xi, \eta \in(0,1)$, either to refine the parameter space or to decrease the continuity at specific knots by multiplication. The knot multiplication is limited to the continuity at the knots because if $C^{-1}$-continuity is reached, the patch becomes split. This is a richer version of h-refinement in conventional BEM, but the user has more control over the geometry other than only splitting the elements into half. Another refinement is the degree elevation which increases the polynomial order of the basis functions. In the degree elevation process, no new unique knots are introduced but the multiplicity at the knots are increased to retain the continuities (or even the discontinuities) at the knots. However, as mentioned above, the number of DOFs introduced in order to increment the polynomial order is much smaller compared to p-refinement in conventional BEM.

After listing the advantages of NURBS based IGA approach over conventional iBEM, some drawbacks also need to be stressed. The biggest drawback of the IGiBEM is that most of the NURBS-based CAD models are not analysis-suitable by default and the pre-processing step can be time consuming. In this paper, the patch interfaces in the numerical illustration will be matching perfectly and watertight. In reality, the industrial CAD files involve many non-matching patch interfaces and trimmed surfaces. However, there are several developments on handling trimmed NURBS patches for industrial applications [26]. Besides, the NURBS models are sometimes too detailed for low-frequency problems, when the wavelength exceeds the characteristic length of a CAD feature. A geometrical simplification might be necessary to obtain fast results within the engineering accuracy levels. However, defeaturing is a risky process where an engineer must consider a loss of accuracy in the simulation results. There might be also a need for pre-processing of the CAD geometry to refine the geometry according to the targeted accuracy and performance of the analysis according to the excitation frequency.

In the next section, the use cases where the NURBS-based IGiBEM is 
advantageous over the conventional BEM will be illustrated with the focus of increasing geometrical complexity.

\section{Numerical illustrations}

In the paper published by Coox et al. [13], IGiBEM is validated against the analytical solution of basic problems. In these problems, a pulsating sphere and plane waves scattered from a rigid sphere are simulated by IGiBEM and the pressure is calculated at directivity field points. Coox et al. demonstrated the advantages of IGiBEM over conventional iBEM in terms of accuracy in those examples. The term, the conventional iBEM, is used for the indirect boundary element models which are isoparametrically discretized by Lagrangian shape functions. The performance of IGiBEM is also demonstrated for more complex geometries. However, the frequency response functions (FRF) were only limited to the low frequencies and only models with a low number of DOFs are simulated due to the performance issues of the in-house MATLAB ${ }^{\circledR}$ [27] code. After pre-processing and system matrix assembly part of the code is translated into $\mathrm{C}++$ via MATLAB MEX API and several code optimizations for efficient calculations are done. This resulted the in-house code to become capable of solving large-scale problems within reasonable time limits. In this section, IGiBEM will not be validated again but will be tested against conventional iBEM software, Siemens PLM Software LMS Virtual.Lab Acoustics v13.7 [28], for several problems with increasing geometrical complexity so as to evaluate the error contributions in a boundary element analysis and to investigate if IGiBEM is able to produce enough improvements to be utilized in acoustic problems of industrial complexity. The performances of the methods are benchmarked by considering the asymptotic complexity of the algorithm. Although the in-house research code is under private license and cannot be shared due to copyright, the results, CAD models and the meshes used in the numerical examples are shared at Zenodo [29].

The sources of errors in the boundary element method can be listed as follows:

- the geometrical error, which is caused by the approximation of the boundary surface,

- the discretization error, which is caused by insufficient polynomial degree or refinement of a mesh to represent the field precisely, 
- the integration error, which is caused by inaccurate integration of the field values over the domain. This occurs due to insufficient quadrature points to represent the polynomial order of the shape functions in Gaussian integration. The integration error is a special case which can affect the post-processing stage as well when integrating the Green's function at the field points.

Many researchers [30, 31, 32] focused on the error analysis on collocation and Galerkin BEM formulations, and how many elements per wavelength must be deployed for different order meshes to ensure a particular accuracy without taking the geometrical accuracy in the direct consideration. However, there is a particular need to assess the importance of geometrical representation in the frequency-domain acoustic simulations, because the wave pattern gets complicated with increasing frequency. The geometrical error is commonly confused with discretization error as the common practice in FEA and BEA is based on the isoparametric approach where the same basis functions are used for geometry and the field, therefore, a better discretization of the domain leads to a better geometrical representation of the boundary surface. To address this issue, the geometrical representation and the field representation must be separated to compare their contribution to the overall error. For this reason, IGiBEM is not only benchmarked against conventional isoparametric iBEM, but also against non-isoparametric iBEM. To eliminate the integration error in the numerical examples, $p+1$ number of Gauss points used for each element, where $p$ is the polynomial degree of the basis function in each direction of the parent element.

The fluid medium is air with a density of, $\rho_{0}=1.225 \mathrm{~kg} / \mathrm{m}^{3}$, and a speed of sound, $c=340 \mathrm{~m} / \mathrm{s}$ for all examples.

\subsection{Vibrating cube}

The first example is chosen to be a geometrically first order model, so both IGiBEM model and iBEM mesh are able to describe the exact representation of the geometry. The main objective here is to eliminate the geometrical errors for iBEM simulations and focus only on the discretization errors at IGiBEM and iBEM in a simple shape. Assume a $0.2 \times 0.2 \times 0.2 \mathrm{~m}^{3}$ cube with a face (in red in Fig. 5) vibrating with an amplitude of unit normal velocity, $\left|v_{n}\right|=1 \mathrm{~m} / \mathrm{s}$, and the other faces are rigid, which means that the normal velocity is equal to zero, $v_{n}=0$. The cube is filled with air and the pressure is calculated at 98 post-processing field points in a $3 \mathrm{D}$ grid formation with 


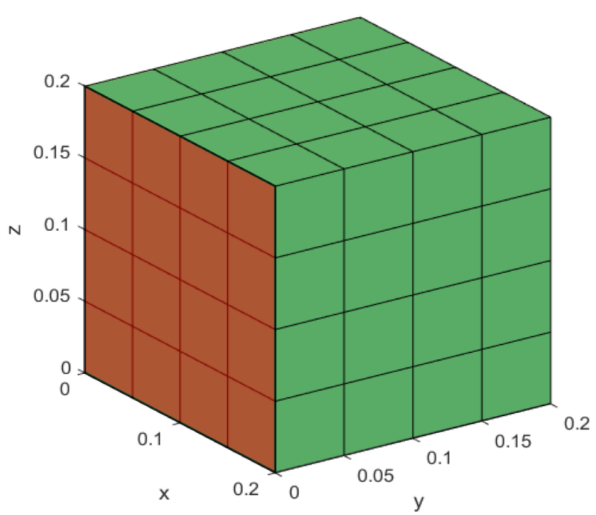

(a) NURBS model

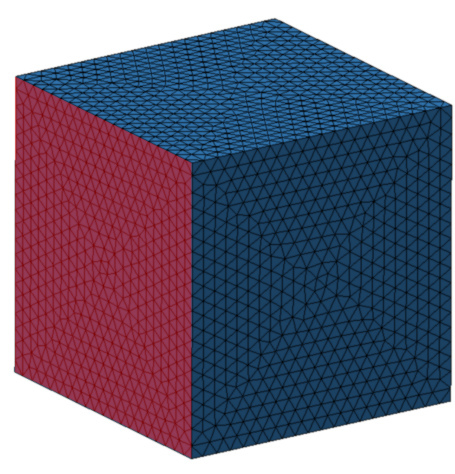

(b) CTRIA3 mesh

Figure 5: Vibrating Cube

an edge length of $0.1 \mathrm{~m}$ inside the cube at the excitation frequencies of $250 \mathrm{~Hz}$ and $3000 \mathrm{~Hz}$ for low and high frequencies, respectively.

The iBEM and IGiBEM results are compared with a reference solution. The reference solution is selected as the Wave Based Method solution, as this method converges to a semi-analytical solution for the bounded acoustical problems which involve geometries in rectangular prism blocks [33]. The $L^{2}$-relative error is calculated by taking the absolute value of the acoustic pressure values at the field points into consideration:

$$
\epsilon=\sqrt{\frac{\sum_{i=1}^{n}\left(\left\|p\left(\mathbf{r}_{i}\right)\right\|-\left\|p_{\text {ref }}\left(\mathbf{r}_{i}\right)\right\|\right)^{2}}{\sum_{i=1}^{n}\left(\left\|p_{\text {ref }}\left(\mathbf{r}_{i}\right)\right\|\right)^{2}}}
$$

where $n$ is the number of field points.

The IGiBEM and iBEM models with different orders and number of elements are numerically tested to evaluate the effect of p-refinement (order elevation) over h-refinement (knot insertion) on the accuracy for simple geometries in low and high-frequency excitations. The highest frequency of the numerical test is $3000 \mathrm{~Hz}$, which corresponds to a wavelength of $113 \mathrm{~mm}$. The coarsest mesh in linear iBEM is $20 \mathrm{~mm}$ elements, which is just at the threshold of six elements per wavelength rule of thumb for linear elements [30]. The iBEM meshes consist of CTRIA3 element type as linear elements and CTRIA6 element type for quadratic elements. The IGiBEM model consists of curvilinear NURBS conforming surface patches. 
The accuracy levels are investigated for p-refined and h-refined models for IGiBEM and iBEM, respectively. When this mesh is p-refined (or the model is degree elevated in the IGiBEM case), the mesh is intact but the polynomial order of the basis functions is increased by one. The h-refinement changes the mesh by dividing the TRIA element into four. Both refinement types quadruple the number of DOFs in the system matrix. As it can be deducted from the Fig. 6, p-refinement/degree-elevation provides a greater increase in accuracy than the h-refinement/knot insertion for both iBEM and IGiBEM.

The convergence of the IGiBEM for the NURBS models with different field orders are presented at low and high frequencies in Fig. 6a, 6b. At low and high frequencies, the order-elevation produces better results than the knot-insertion without increasing the polynomial order for the IGiBEM solutions.

At low frequencies, the p-refinement reduce the error by factor of 10 for a cost of quadruple DOFs, see Fig. 6c. This means 64 times slower simulation for such accuracy improvement. However, the same p-refinement leads to almost decrease by factor of $10^{2}$ in error at a high frequency, see Fig. 6d, Therefore, it is logical to assume that p-refinement pays-off the computational cost at high frequencies, where the field gets complicated.

So far, no geometrical error was involved. The influence of h-refinement and p-refinement on reducing the discretization error is tested and it is shown that h-refinement has less impact than p-refinement on discretization error when the geometry is neglected, as expected from the theory [34]. In the next examples, the role of the geometrical error is also investigated.

\subsection{Car hood}

A sports car hood is chosen as a more complex model with a geometrical order of two $(p=2)$ in this example. The outer box dimensions of the model are roughly $1.8 m \times 1.4 m \times 0.4 m$ and the model consists of 14 conforming patches. In this case, the car hood vibrates with a unit panel velocity, $\left(\left|v_{n}\right|=\right.$ $1 \mathrm{~m} / \mathrm{s}$ ), and the sound radiates through the unbounded acoustic domain. The pressure values are evaluated in a cloud of 218 uniformly distributed field points placed on a spherical surface $1 \mathrm{~m}$ away from the center of gravity of the structure.

This geometry is a perfect example of an open-boundary surface. The performance of IGiBEM is again benchmarked against the conventional and non-isoparametric iBEMs. However, this time the geometry is arbitrary and 


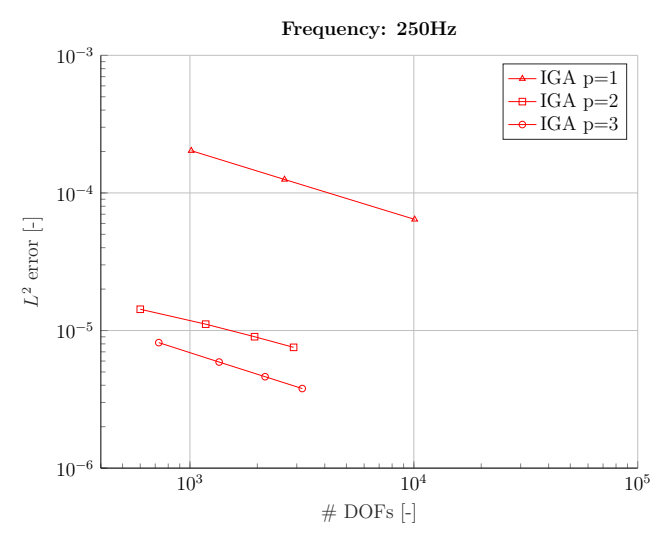

(a) IGA in low frequency

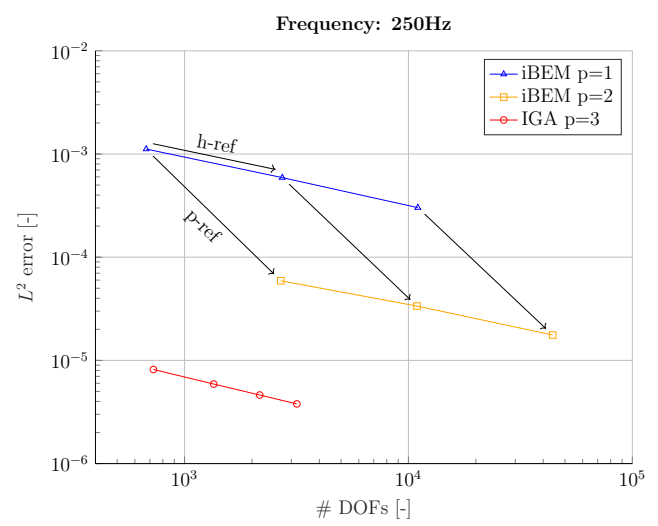

(c) iBEM results in low frequency

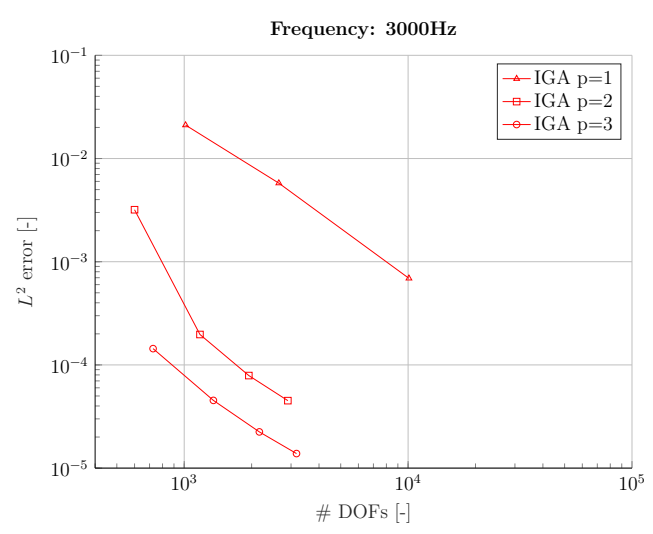

(b) IGA in high frequency

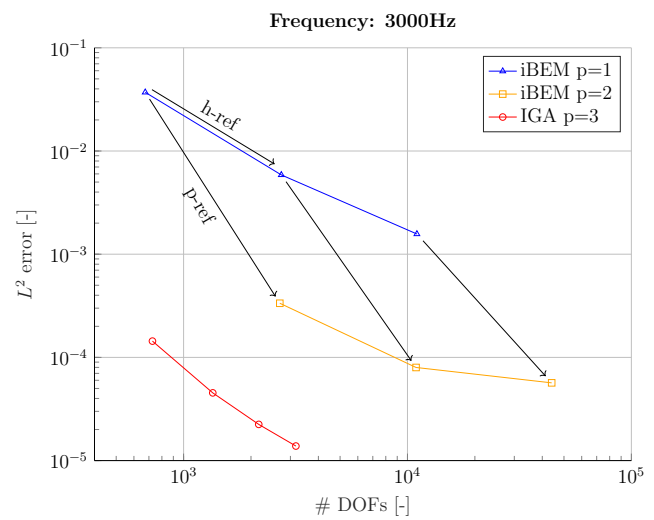

(d) iBEM results in high frequency

Figure 6: The comparison of IGiBEM and iBEM results for the vibrating cube example

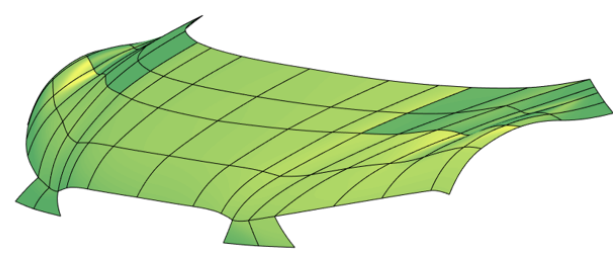

(a) NURBS model

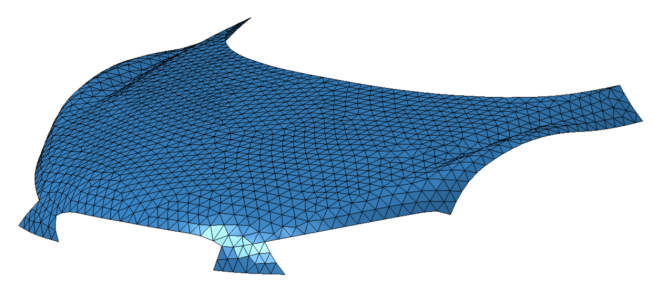

(b) CTRIA3 mesh

Figure 7: Car hood models 
there is no analytical solution for the specified problem. Therefore, it is necessary to define a reference model first. In order to define the reference model, the results of models which are solved by different methods are benchmarked against each other with a cross-referencing scheme. In the cross-referencing scheme, the methods are compared to each other by calculating the error values of the iBEM models with respect to the most refined IGiBEM models, and vice versa. The $L^{2}$ relative-error of the pressure values at the field points are taken as the measure of convergence. There are three IGiBEM models with $2 \mathrm{k}, 5 \mathrm{k}, 20 \mathrm{k}$ DOFs, three linear iBEM models with $1.3 \mathrm{k}, 5 \mathrm{k}$, and $20 \mathrm{k}$ DOFs and two quadratic iBEM models with $5 \mathrm{k}$ and $20 \mathrm{k}$ DOFs. The convergence rates of the methods are benchmarked against each other by taking the solution of the most refined model of each method as the reference within a frequency range of $[100,500] \mathrm{Hz}$, see Fig. 8. IGiBEM solutions does not converge neither to linear iBEM model with 20k DOFs, see Fig. 8a, nor to the quadratic iBEM model with 20k DOFs, see Fig 8b, while linear and quadratic iBEM solutions converge to the solution of the IGiBEM model with 20k DOFs by increasing DOFs, see Fig. 8c. As a result, the solution of the most refined IGiBEM model is found to be the most accurate solution and taken as the reference solution for this problem.

After the determination of the reference, the geometrical influence on the overall error is investigated to distinguish the contribution of geometrical error from the discretization error. In order to make this distinction, a non-isoparametric approach is introduced to the iBEM. By means of a non-isoparametric approach, the mesh can be kept intact while the field order is elevated. Therefore, two linear iBEM models (with 1.3k, 5k DOFs) are selected as the bases for the non-isoparametric iBEM for this numerical experiment. The field order is increased from linear to quadratic by introducing second-order Lagrangian shape functions, therefore, adding nodes without changing the linear mesh. The simulations with non-isoparametric iBEM are also run in the same frequency range and benchmarked against the linear and quadratic iBEM by taking the IGiBEM solution as reference. The relative error values show that non-isoparametric iBEM acquired from a linear iBEM model (base model) gives more accurate results than a linear mesh using half the element size from 100 to $500 \mathrm{~Hz}$, see in Fig. 9a. This shows that even the increase in the field order while keeping the geometry identical yields more accurate results than h-refined linear mesh at low frequencies, even though the geometry is better represented by the h-refined linear mesh. 


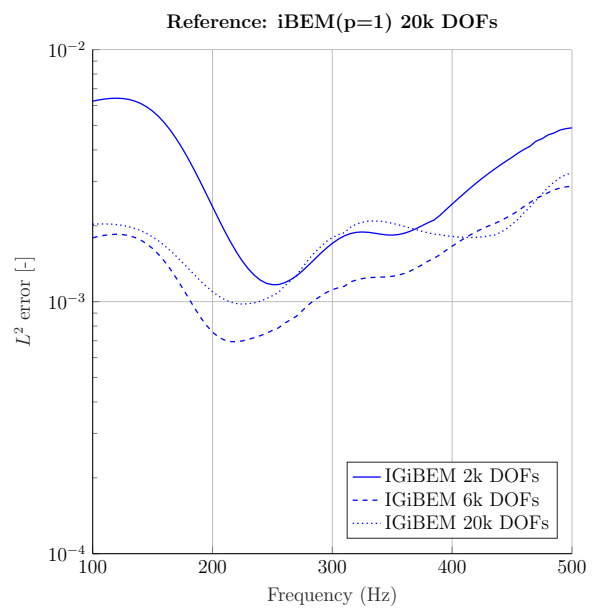

(a) IGiBEM convergence against the finest linear iBEM solution

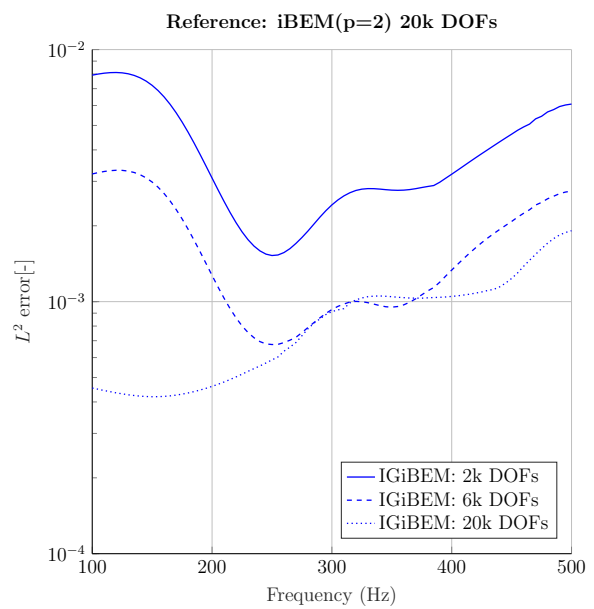

(b) IGiBEM convergence against the finest quadratic iBEM solution

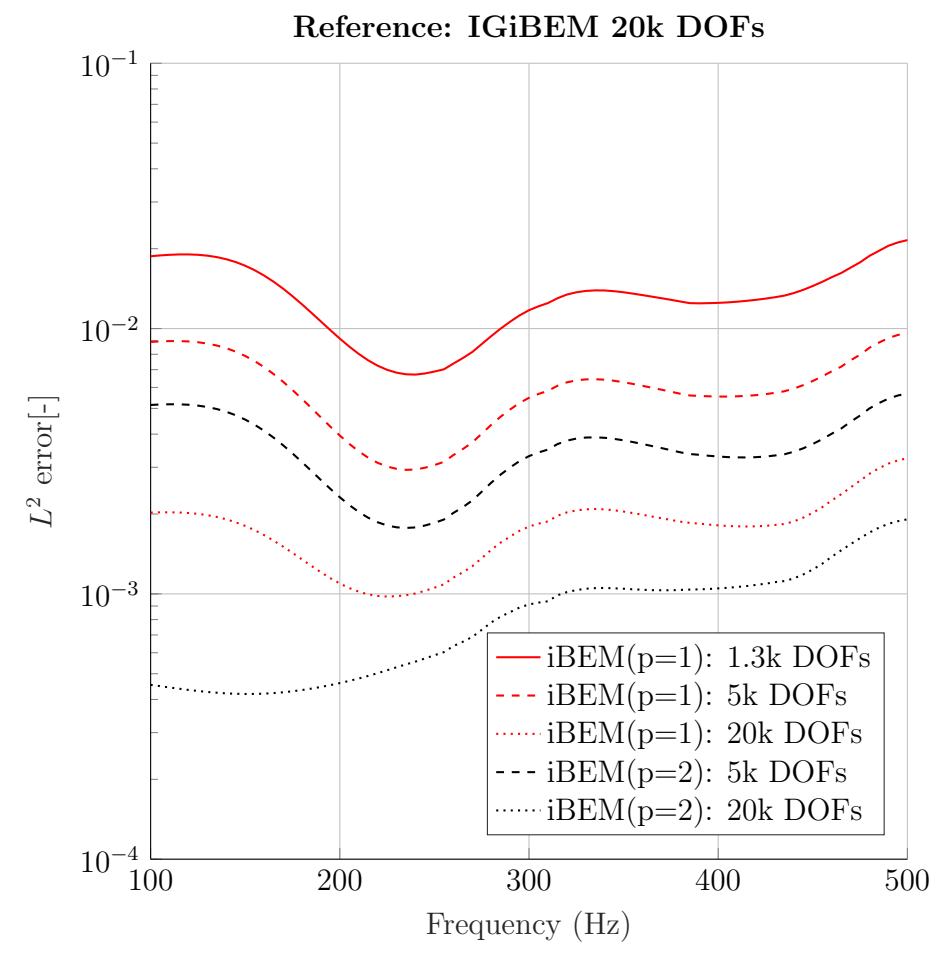

(c) linear and quadratic iBEM convergences against the finest IGiBEM solution

Figure 8: The convergence plots of the IGiBEM and iBEM $(p=1,2)$ models with cross-referencing scheme for the car hood problem 


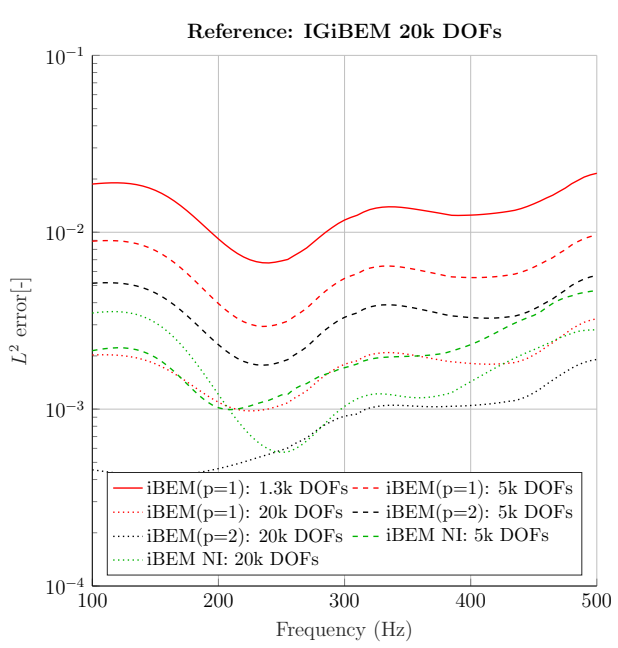

(a) $L^{2}$ relative error with respect to the $\operatorname{IGiBEM}(\mathrm{p}=2) 20 \mathrm{k}$ DOFs from $100 \mathrm{~Hz}$ to $500 \mathrm{~Hz}$

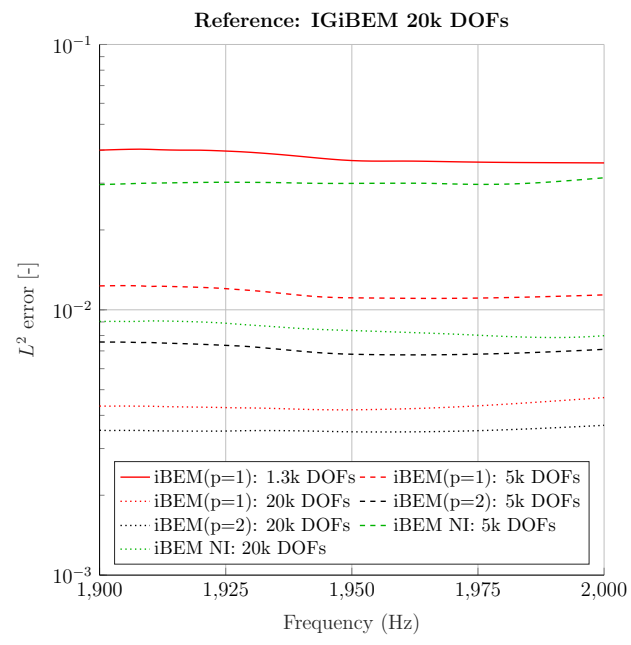

(b) $L^{2}$ relative error with respect to the $\operatorname{IGiBEM}(\mathrm{p}=2) 20 \mathrm{k}$ DOFs from $1900 \mathrm{~Hz}$ to 2000 $\mathrm{Hz}$

Figure 9: Estimates of discretization and geometrical errors for the car hood example at low and high frequencies

The problem gets more complicated when the vibration frequency is higher due to the wave-based nature of the problem. When the methods are numerically tested for the same problem but at higher frequencies, the geometrical accuracy of the model becomes more prominent. As one can see in Fig. 9b, the non-isoparametric BEM does not perform better than the iBEM solution of an h-refined mesh in terms of accuracy. This means that an increase of field order is not sufficient to maintain the accuracy level at higher frequencies. The geometrical accuracy of the model gains in importance as compared to how the field or wave is discretized at high frequencies for acoustic problems.

\subsection{Loudspeaker}

The last numerical example is a $0.3 \times 0.5 \times 0.45 \mathrm{~m}^{3}$ loudspeaker with a woofer, highlighted in red in Fig. 10, vibrating with unit normal velocity $\left(\left|v_{n}\right|=1 \mathrm{~m} / \mathrm{s}\right)$ whereas the rest of the surface model is rigid $\left(v_{n}=0 \mathrm{~m} / \mathrm{s}\right)$. The geometrical order of the CAD model is formed by cubic NURBS functions, $p=3$. The shape of the loudspeaker has features such as the woofer, twitter and the porthole, and the velocity boundary condition is applied to a rounded part of the boundary surface. These elaborate features and 


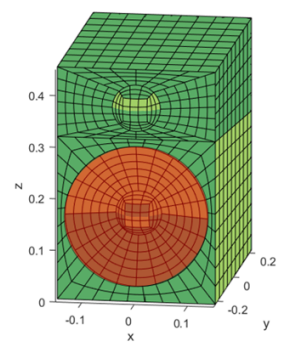

(a) NURBS model
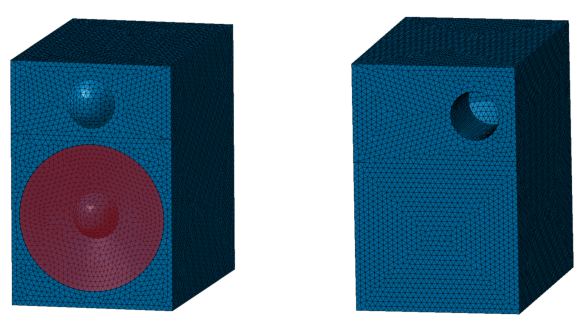

(b) CTRIA3 mesh

Figure 10: Loudspeaker models

boundary conditions make this model topologically the most complicated shape among the numerical examples presented in this paper. Moreover, the loudspeaker model is an almost-closed shape which makes the wave pattern scatter multiple times from the inner walls of the structure. The porthole naturally connects the inner and outer fluid domains and makes the loudspeaker surface an open-boundary. The CAD model consists of 37 patches with non-conforming interfaces.

Due to the high geometrical order, a low number of DOFs could be used in the IGiBEM model. Therefore, the base IGiBEM model is selected to be a small model with 2763 DOFs. The conventional linear iBEM model is selected to be a larger model with 10k DOFs consisting of CTRIA3 elements. The element size of the iBEM model is $10 \mathrm{~mm}$. As the highest frequency solved for in this numerical example is $2000 \mathrm{~Hz}$, the six element per wavelength rule is satisfied for linear elements.

The sound pressure values are calculated at the 120 directivity field points (with $3^{\circ}$ angular distance) which are placed $1 \mathrm{~m}$ away from the center of the loudspeaker cabinet. The frequency response function at a single field point is illustrated in Fig. 11. The directivity plot of the sound pressure postprocessed at the field points at 500 and $2000 \mathrm{~Hz}$ is visualized in Fig. 12 . Both figures show that the solutions match over a large range of frequency. However, a reference model is again to be determined in order to make an error analysis. For this reason, the refined versions of the iBEM and IGiBEM models are simulated.

Again the cross-referencing scheme used in the previous example is applied in order to determine the reference solution because there is no analytical or experimental solution to the associated problem. It is concluded 


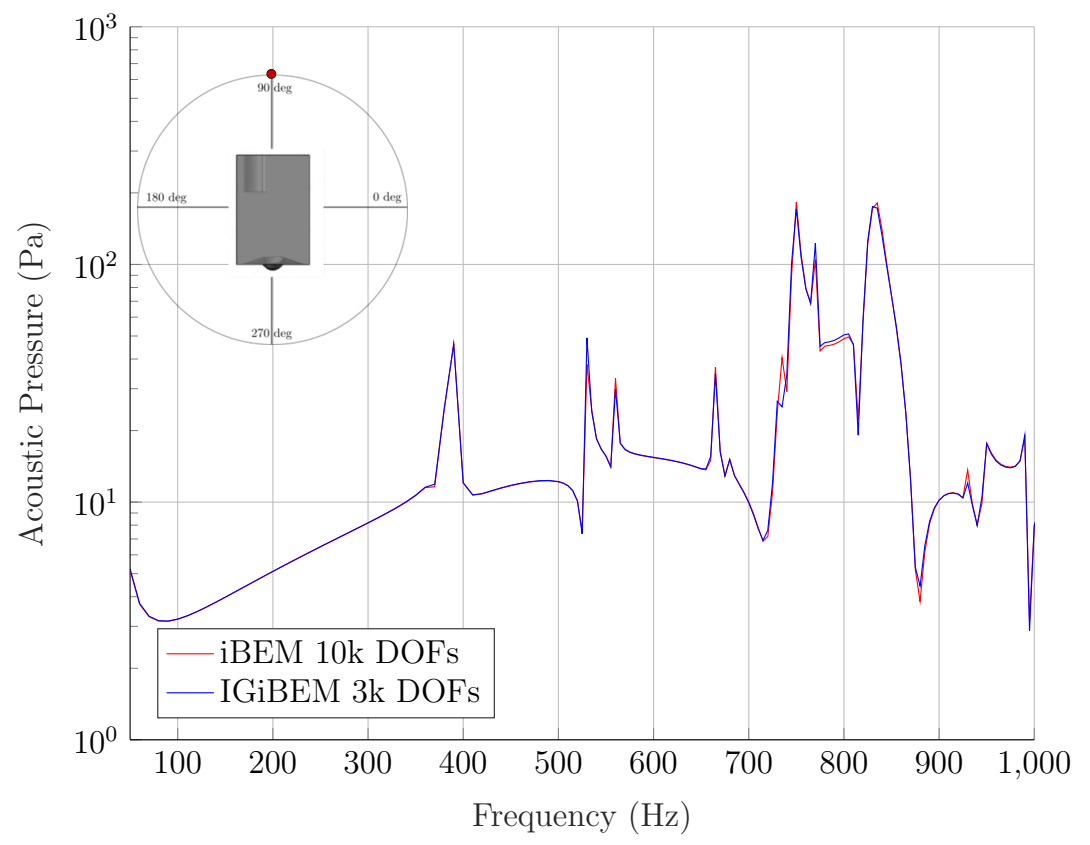

(a) at low frequencies

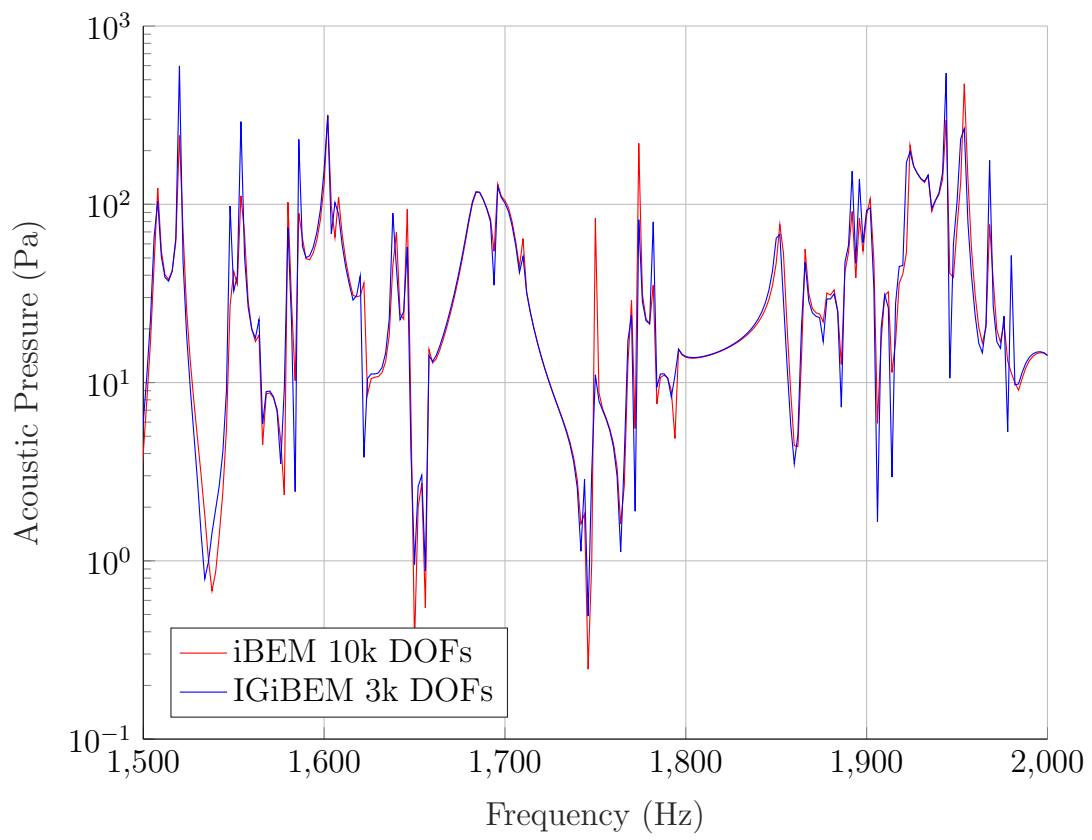

(b) at high frequencies

Figure 11: Frequency response function of the acoustic pressure in $\mathrm{Pa}$ at a field point at the back of the loudspeaker for iBEM and RABBEM solutions 


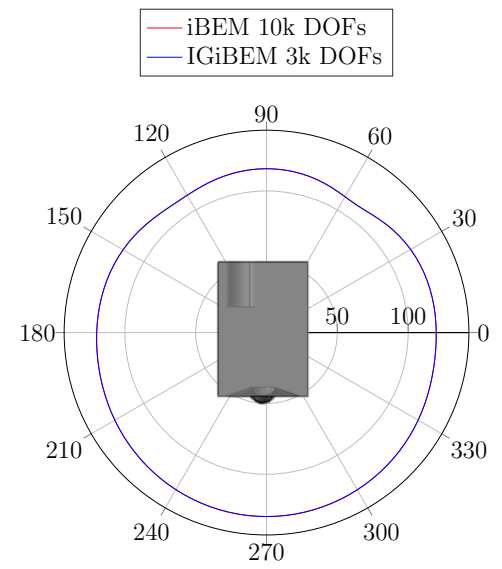

(a) Sound Pressure at $500 \mathrm{~Hz}($ in $\mathrm{dB})$

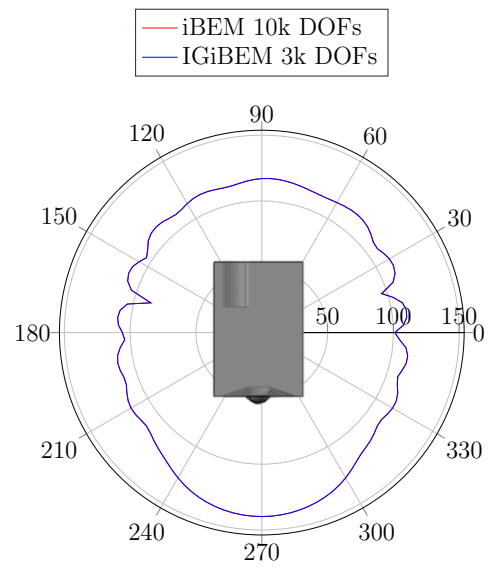

(b) Sound Pressure at $2000 \mathrm{~Hz}$ (in dB)

Figure 12: Directivity plots at low and high frequencies

from Fig. 13 that the IGiBEM solution is more accurate compared to iBEM $(p=1,2)$ solutions. As one can see, the IGiBEM does not converge to the solution of the most refined iBEM model in frequency regions close to $500 \mathrm{~Hz}$, see Fig. 13a $13 \mathrm{~b}$. However, both iBEM solutions show better convergence to the results of the finest IGiBEM model in frequency range from 50 to $500 \mathrm{~Hz}$, see Fig. 13c. Therefore, we select again the solution with the finest IGiBEM model as the reference solution for this numerical example.

The influence of the geometrical representation on overall error is again investigated by adding the non-isoparametric iBEM with linear mesh but quadratic Lagrangian shape functions to the comparison of the FRFs. However, in this numerical example, none of the conventional iBEM meshes in comparison, even the quadratic one with CTRIA6 elements, represents the exact geometry as the geometrical order of the loudspeaker model is higher than two. Therefore, the geometrical error is not overcome entirely, but the improvements on the accuracy with increasing geometrical and/or discretization order are.

The $L^{2}$-errors over the pressure values at the field points are calculated for the conventional iBEM simulations with linear $(\mathrm{p}=1)$ and quadratic $(\mathrm{p}=2)$ surface meshes and the non-isoparametric iBEM. In this example, iBEM $(\mathrm{p}=1)$ simulation with $10 \mathrm{k}$ DOFs and non-isoparametric simulation with $40 \mathrm{k}$ DOFs share the same geometrical representation, but the non-isoparametric model has additional DOFs at the middle of the element edges. The con- 


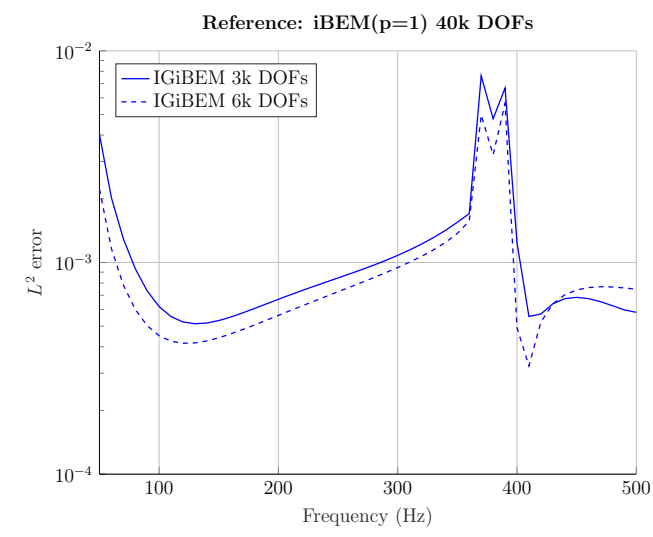

(a) IGiBEM results with respect to iBEM $(p=1)$ 40k DOFs solution

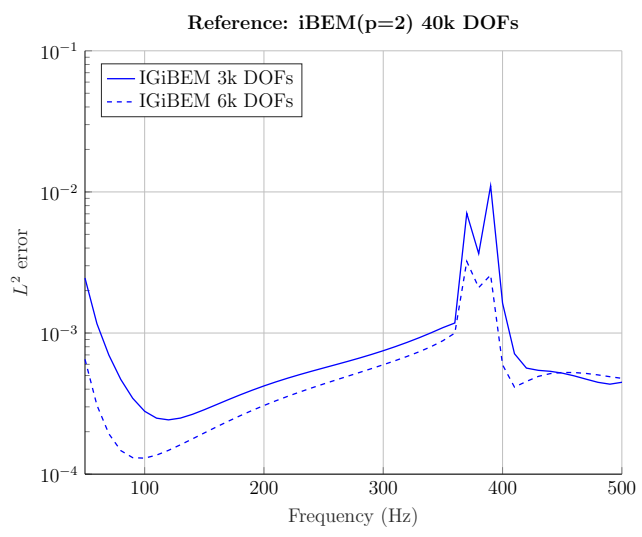

(b) IGiBEM results with respect to iBEM $(\mathrm{p}=2)$ 40k DOFs solution

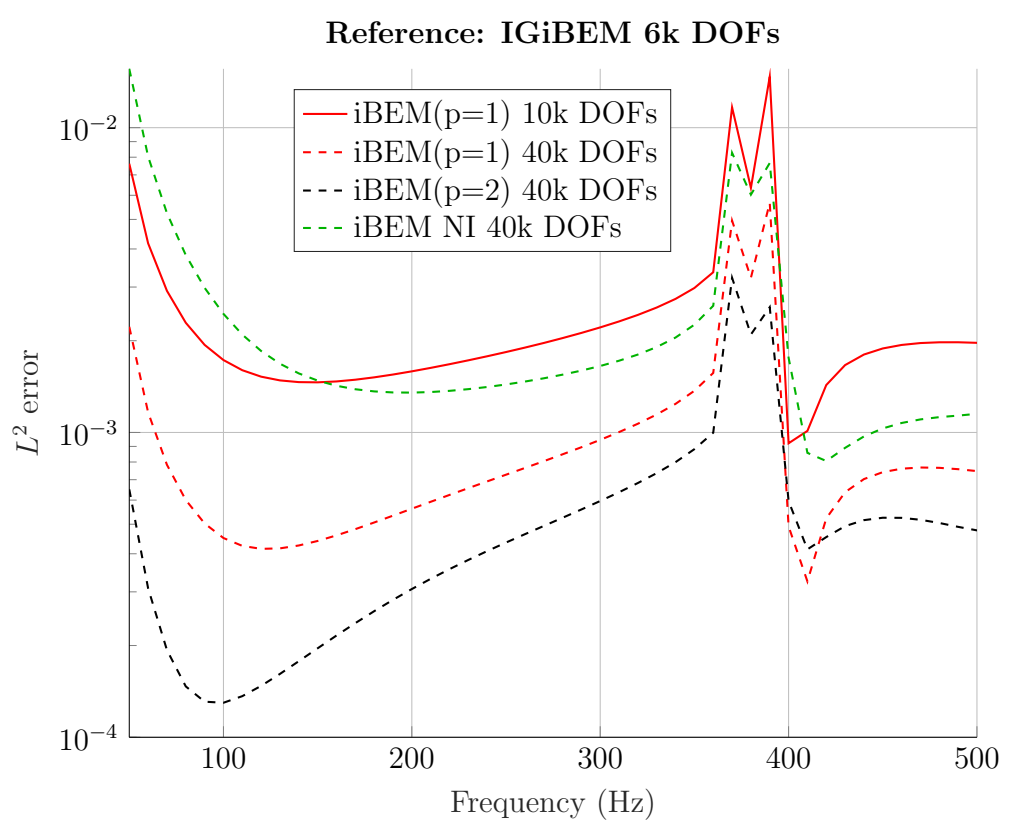

(c) iBEM results with respect to IGiBEM ( $\mathrm{p}=3) 6 \mathrm{k}$ DOFs solution

Figure 13: The convergence plots of the IGiBEM and iBEM $(p=1,2)$ models with cross-referencing scheme for the loudspeaker problem 
ventional iBEM models $(\mathrm{p}=1,2)$ with $40 \mathrm{k}$ DOFs are derived from the iBEM 10k model by h- and p-refinement, respectively. One can notice that the linear iBEM 40k DOFs model performs better than the non-isoparametric 40k DOFs model which has a better discretization but worse geometrical accuracy. This shows that the geometrical error dominates the discretization error even at low frequencies for a complex case.

\section{Conclusion}

The results show that a correct geometrical representation is critical for industrial acoustics problems. Engineers using conventional iBEM try to overcome this issue by creating a new mesh for each frequency range to comply with the six-elements per wavelength rule of thumb for linear elements. However, this assumption is made only by taking the discretization of the acoustic pressure field into consideration and linear elements are typically not ideal for representing curved geometries. The presented numerical studies demonstrate that the geometrical error can become a bigger contributor to the overall accuracy than the discretization error for complex problems. The importance of an accurate geometrical representation grows with increasing frequency of the excitation due to the wave-based nature of the problem. The modeling efficiency of the presented method enables the pre-processing of the model with ease for changing frequencies. It is very flexible with h- and p-refinement to reduce the discretization errors and it never suffers from geometrical errors. Therefore, the IGiBEM shows a great potential for getting more accurate results in a large variety of acoustics problems. In addition, the computational cost reduction obtained by using the isogeometric approach is significant. As it can be deduced from the loudspeaker example, an IGiBEM model with 6k DOFs shows a better performance than 40k DOFs iBEM models both in efficiency and accuracy. As the system matrix solution with an asymptotic complexity of $O\left(N^{3}\right)$ determines the computational cost, the simulation time improves around 300 times.

\section{Acknowledgements}

This research is funded by Marie Curie Initial Training Network(ITN) BATWOMAN - Basic Acoustics Training \& Workprogram On Methodologies for Acoustics - Network (PITN-GA-605867). The Research Fund KU Leuven is gratefully acknowledged for its support. The research of E. Deckers is 
funded by a postdoctoral grant from the Research Foundation - Flanders (FWO). 


\section{References}

[1] T. J. Hughes, J. A. Cottrell, Y. Bazilevs, Isogeometric analysis: Cad, finite elements, nurbs, exact geometry and mesh refinement, Computer methods in applied mechanics and engineering 194 (2005) 4135-4195.

[2] Y. Chen, J. Lee, A. Eskandarian, Meshless methods in solid mechanics, Springer Science \& Business Media, 2006.

[3] H. Sakurai, Element-free methods vs. mesh-less cae, International Journal of Computational Methods 3 (2006) 445-464.

[4] S. Fernández-Méndez, A. Huerta, Imposing essential boundary conditions in mesh-free methods, Computer methods in applied mechanics and engineering 193 (2004) 1257-1275.

[5] J. A. Cottrell, A. Reali, Y. Bazilevs, T. J. Hughes, Isogeometric analysis of structural vibrations, Computer methods in applied mechanics and engineering 195 (2006) 5257-5296.

[6] N. Nguyen-Thanh, J. Kiendl, H. Nguyen-Xuan, R. Wüchner, K. Bletzinger, Y. Bazilevs, T. Rabczuk, Rotation free isogeometric thin shell analysis using pht-splines, Computer Methods in Applied Mechanics and Engineering 200 (2011) 3410-3424.

[7] V. P. Nguyen, C. Anitescu, S. P. Bordas, T. Rabczuk, Isogeometric analysis: an overview and computer implementation aspects, Mathematics and Computers in Simulation 117 (2015) 89-116.

[8] I. Temizer, P. Wriggers, T. Hughes, Three-dimensional mortar-based frictional contact treatment in isogeometric analysis with nurbs, Computer Methods in Applied Mechanics and Engineering 209 (2012) 115128.

[9] L. De Lorenzis, I. Temizer, P. Wriggers, G. Zavarise, A large deformation frictional contact formulation using nurbs-based isogeometric analysis, International Journal for Numerical Methods in Engineering 87 (2011) $1278-1300$.

[10] P. N. Nielsen, A. R. Gersborg, J. Gravesen, N. L. Pedersen, Discretizations in isogeometric analysis of navier-stokes flow, Computer methods in applied mechanics and engineering 200 (2011) 3242-3253. 
[11] Y. Bazilevs, V. M. Calo, Y. Zhang, T. J. Hughes, Isogeometric fluid-structure interaction analysis with applications to arterial blood flow, Computational Mechanics 38 (2006) 310-322.

[12] R. N. Simpson, M. A. Scott, M. Taus, D. C. Thomas, H. Lian, Acoustic isogeometric boundary element analysis, Computer Methods in Applied Mechanics and Engineering 269 (2014) 265-290.

[13] L. Coox, O. Atak, D. Vandepitte, W. Desmet, An isogeometric indirect boundary element method for solving acoustic problems in openboundary domains, Computer Methods in Applied Mechanics and Engineering 316 (2017) 186-208.

[14] L. Coox, E. Deckers, D. Vandepitte, W. Desmet, A performance study of nurbs-based isogeometric analysis for interior two-dimensional timeharmonic acoustics, Computer Methods in Applied Mechanics and Engineering 305 (2016) 441-467.

[15] N. Atalla, F. Sgard, Finite Element and Boundary Methods in Structural acoustics and vibration, CRC Press, 2015.

[16] N. A. Gumerov, R. Duraiswami, Fast multipole methods for the Helmholtz equation in three dimensions, Elsevier, 2005.

[17] B. Marussig, J. Zechner, G. Beer, T.-P. Fries, Fast isogeometric boundary element method based on independent field approximation, Computer Methods in Applied Mechanics and Engineering 284 (2015) 458488.

[18] L. Banjai, W. Hackbusch, Hierarchical matrix techniques for low-and high-frequency helmholtz problems, IMA journal of numerical analysis 28 (2007) 46-79.

[19] T. Wu, Boundary element acoustics: fundamentals and computer codes, volume 7, Wit Pr/Computational Mechanics, 2000.

[20] S. A. Sauter, C. Schwab, Boundary element methods, Springer, 2010.

[21] L. Piegl, W. Tiller, The NURBS book, Springer Science \& Business Media, 2012. 
[22] M. G. Cox, The numerical evaluation of b-splines, IMA Journal of Applied Mathematics 10 (1972) 134-149.

[23] C. De Boor, On calculating with b-splines, Journal of Approximation theory 6 (1972) 50-62.

[24] M. Spink, Nurbs toolbox, Matlab Central [online], Accessed on June 20 (2018).

[25] L. Coox, F. Greco, O. Atak, D. Vandepitte, W. Desmet, A robust patch coupling method for nurbs-based isogeometric analysis of nonconforming multipatch surfaces, Computer Methods in Applied Mechanics and Engineering 316 (2017) 235-260.

[26] B. Marussig, T. J. Hughes, A review of trimming in isogeometric analysis: Challenges, data exchange and simulation aspects, Archives of computational methods in engineering (2017) 1-69.

[27] MATLAB, version 9.4.0 (R2018a), The MathWorks Inc., Natick, Massachusetts, 2018.

[28] S. P. S. L. V. Acoustics, version 13.7, https://www.plm. automation.siemens.com/global/en/products/simulation-test/ acoustic-simulation.html, 2014. [Online; accessed on 24-07-2019].

[29] E. O. Inci, L. Coox, O. Atak, E. Deckers, W. Desmet, The dataset for the isogeometric boundary element method for acoustic problems, https://zenodo.org/record/3349905\#.XThyxOgzaUk, 2019. [Online; pushlished on 24-July-2019].

[30] S. Marburg, Six boundary elements per wavelength: is that enough?, Journal of Computational Acoustics 10 (2002) 25-51.

[31] G. Hsiao, R. Kleinman, Error analysis in numerical solution of acoustic integral equations, International Journal for Numerical Methods in Engineering 37 (1994) 2921-2933.

[32] E. Perrey-Debain, J. Trevelyan, P. Bettess, Wave boundary elements: a theoretical overview presenting applications in scattering of short waves, Engineering Analysis with Boundary Elements 28 (2004) 131-141. 
[33] B. Van Genechten, O. Atak, B. Bergen, E. Deckers, S. Jonckheere, J. S. Lee, A. Maressa, K. Vergote, B. Pluymers, D. Vandepitte, et al., An efficient wave based method for solving helmholtz problems in threedimensional bounded domains, Engineering Analysis with Boundary Elements 36 (2012) 63-75.

[34] P. Solin, K. Segeth, I. Dolezel, Higher-order finite element methods, Chapman and Hall/CRC, pp. 313-372. 